Article

\title{
Fast and Simple Fabrication of Superhydrophobic Coating by Polymer Induced Phase Separation
}

\author{
Yu-Ping Zhang ${ }^{1, *}$, Pan-Pan Li ${ }^{1}$, Peng-Fei Liu ${ }^{1}$, Wan-Qing Zhang ${ }^{1}$, Ji-Chao Wang ${ }^{1}$, \\ Cheng-Xing Cui ${ }^{1,2}$, Xiang-Jun $\mathrm{Li}^{3}$ and Ling-Bo $\mathrm{Qu}^{2, *}$ \\ 1 Henan Institute of Science and Technology, Xinxiang 453000, China; ccxcyh@gmail.com (P.-P.L.); \\ lpf8856@163.com (P.-F.L.); zhangwqzzu@163.com (W.-Q.Z.); wangjichao@hist.edu.cn (J.-C.W.); \\ chengxingcui@hist.edu.cn (C.-X.C.) \\ 2 College of Chemistry and Molecular Engineering, Zhengzhou University, Henan 450001, China \\ 3 School of Chemical Sciences, University of Chinese Academy of Sciences, Beijing 100049, China; \\ lixiangj@ucas.ac.cn \\ * Correspondence: zhangyuping@hist.edu.cn (Y.-P.Z.); qulingbo@zzu.edu.cn (L.-B.Q.); \\ Tel.: +86-373-304-0926 (Y.-P.Z.)
}

Received: 13 January 2019; Accepted: 5 March 2019; Published: 11 March 2019

check for updates

\begin{abstract}
Polymerization-induced phase separation is comparatively applied to fabricating a superhydrophobic micro/nano surface by microwave and ultraviolet (UV) irradiation. The monolithic coatings can be tailored easily on different substrates with excellent chemical and mechanical stability in rigid conditions. Importantly, the patterned filter paper is successfully used for the colorimetric detection of nitrite handily.
\end{abstract}

Keywords: polymerization; superhydrophobic material; microwave irradiation; UV irradiation; nitrite

\section{Introduction}

Wetting phenomena of solid surface are ubiquitous in nature as well as in many commercial applications including oil/water separation, membrane engineering and microfluidics [1-5]. Surface with a static water contact angle (WCAs) greater than $150^{\circ}$ and roll-off angles (RAs) below $10^{\circ}$ for water is generally defined as superhydrophobic one [6,7]. Numerous approaches have been attempted to fabricate superhydrophobic materials, which include chemical/mechanical etching [8], sol-gel process [9], layer-by-layer self-assembly [10], electrospinning [11], electrodeposition and CVD (chemical vapor deposition) [12,13]. Unfortunately, the preparation procedures are usually complicated and time-consuming, not to mention their dependence on multi-step procedures or some special instruments as well as post-modification by expensive chemicals with low surface energy. Especially, weak adhesion between the surfaces and the substrates usually impedes their practical application. Therefore, it is necessary to find more convenient methods and select suitable materials for fabricating superhydrophobic coatings [14-16]. Monolithic materials have been widely used as the absorbent in sample pretreatment and the stationary phase in HPLC, $\mu$-HPLC and capillary electrochromatography (CEC) due to their unique advantages such as fast separation, high-linear flow velocities and nonrequirement of frits [17]. It is well known that the stationary phases in reverse phase liquid chromatography (RPLC) are common hydrophobic materials but very few literatures are reported how to modify the hydrophobic stationary phase to superhydrophobic one [18-20]. 
Levkin and co-workers prepared superhydrophobic coatings of poly (butyl methacrylateco-ethylene dimethacrylate, BMA-EDMA) and poly(styrene-co-1,4-divinylbenzene, ST-DVD) but thermally initiated polymerization was carried out at a temperature of $70{ }^{\circ} \mathrm{C}$ for $24 \mathrm{~h}$ and a detailed investigation of ruggedness was not considered [19]. Afterwards, Kato and Sato reported a ultraviolet (UV) triggered phase-separation process to convert a coated precursor to superhydrophobic coatings but soluble linear polymers should be added to the porogenic agents for the process of polymerization-induced phase separation (PIPS) [20]. Afterwards, microporous poly(BMA-co-EDMA) surfaces were prepared via UV-initiated free-radical polymerization. Subsequent infusion of fluorocarbon lubricants into the porous microtexture resulted in liquid-repellent slippery surfaces with a marine antibiofouling property [21].

In the present communication, we focus on a more simple, rapid and versatile approach to modify hydrophobic polymer monoliths to the superhydrophobic one under an open-air environment. For the first time, PIPS is carried out through the optimal selection of monomer and porogen with the help of microwave and UV irradiation (see Scheme S1). The fabrication of superhydrophobic monoliths take only $4 \mathrm{~min}$ for microwave-curing method. The operation includes three steps: (1) Dropping the polymerization mixture on a substrate, (2) Microwave or UV irradiation to induce phase separation and (3) Ethanol flushing the porous monolithic surface. By optimizing the appropriate composition of the porogenic agents, the micro- and nanoscale superhydrophobic surface is logically tailored.

\section{Materials and Methods}

Butyl methacrylate (BMA), ethylene dimethacrylate (EDMA), methacrylic acid (MAA), 3-(methacryloyloxy)propyl]trimethoxysilane ( $\gamma$-MPTMS), ethanol, isooctane, toluene, butanediol, lauryl alcohol, azobisisobutyronitrile (AIBN) used as a thermal initiator are purchased from Aladdin Chemical Reagent Co., Ltd. (Shanghai, China) and Tianjin Kermel Chemical Reagent Co., Ltd., (Tianjin, China). Irgacure 1800 as a UV photo-initiator is donated by Ciba-Geigy (Tianjing, China).

The glass slide is pretreated to create binding sites for the attachments of the polymeric materials. Firstly, the glass slide is immersed in $1 \mathrm{M} \mathrm{NaOH}$ for $30 \mathrm{~min}$ and then with $0.1 \mathrm{M} \mathrm{HCI}$ for $30 \mathrm{~min}$. Subsequently, it is flushed with $\mathrm{H}_{2} \mathrm{O}$ for several times and then dried by passing nitrogen gas. The glass plates are immersing in a silanizing solution, a solution of $50 \%(v / v)$ of $\gamma$-MPTMS in ethanol overnight at $60^{\circ} \mathrm{C}$, then dried for the next use. The purpose of surface pretreatment is to increase the concentration of surface silanol groups. Since silanol groups on the glass surface represent the principal binding sites for in situ created polymer-based monolith, higher concentration of these binding sites on the glass surface would facilitate the coating formation of highly secured organic-based monolith [18-20]. Microwave irradiation is carried out in a simple home microwave oven with a common frequency of 2450 HZ. An XL-1500 UV cross-linker (Spectronics Corp., Westbury, NY, USA) is equipped with six $15 \mathrm{~W}$ blacklight tubes, in which the dropping surfaces of different substrates are irradiated with a total dose of $0.9 \mathrm{~J} / \mathrm{cm}^{2}$ at a wavelength of $365 \mathrm{~nm}$.

Briefly, a polymerization solution consisting of monomer (BMA or MAA), crosslinker (EDMA), porogenic mixtures and a thermal initiator (AIBN) or a UV photo-initiator (Irgacure 1800) is spread evenly on a substrate such as the glass slide, filter paper, $\mathrm{Cu}$ foil, $\mathrm{Al}$ foil and PTFE film, then irradiated by microwave or UV. The amount of both initiators are about $1.0 \%(w / v)$ of the used monomers. In the PIPS process, the solvents are miscible with the cross-linkable monomers but immiscible with the generating network polymers, so that a phase-separated state can be obtained. After flushing off unreacted compositions, a monolithic surface with a defined wettability and ruggedness is formed. Illustration of the fabrication procedures and relative mechanism is shown in Scheme 1. 


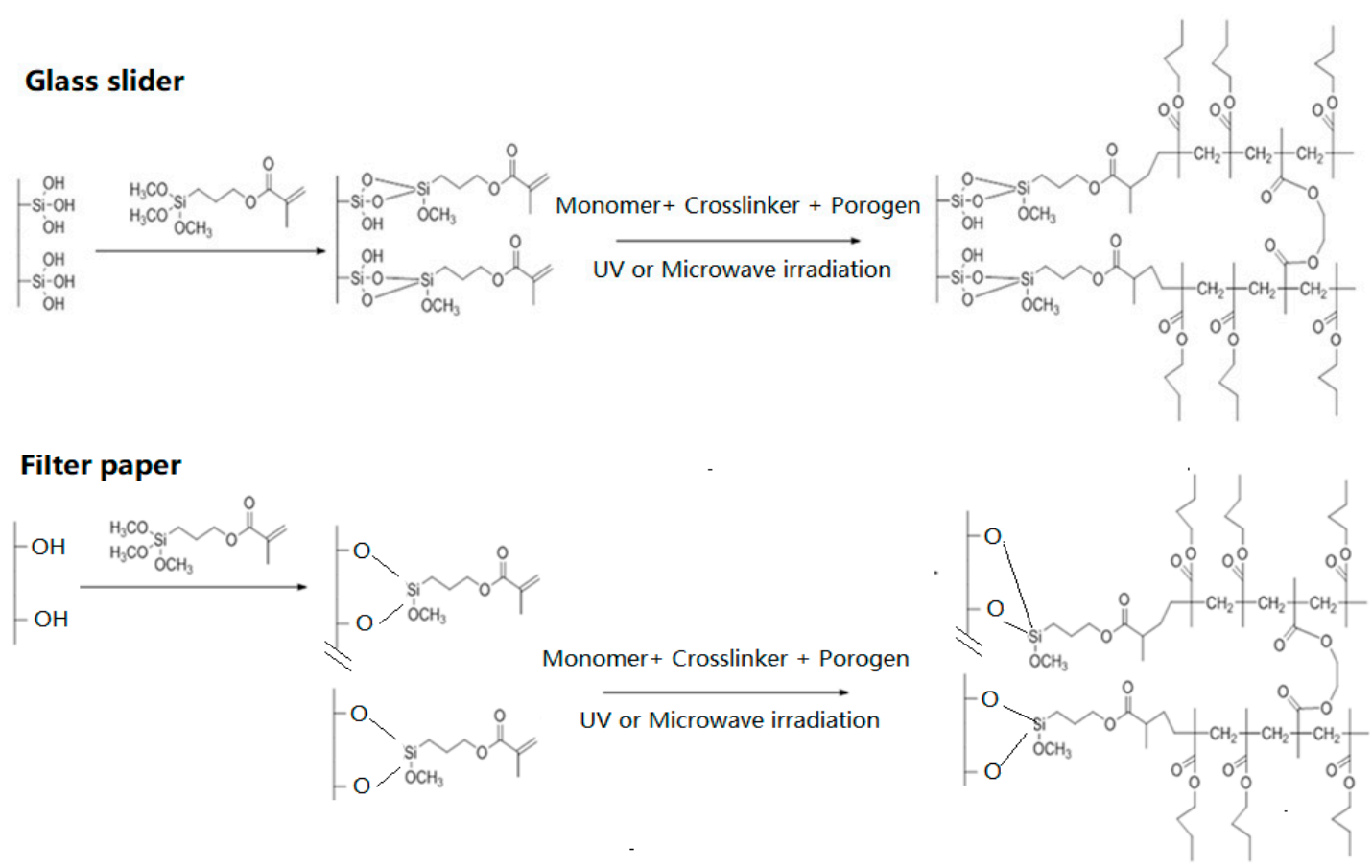

Scheme 1. Illustration of the fabrication procedures and relative mechanism on the glass slide and filter paper.

\section{Results and Discussion}

\subsection{Preparation of the Monolithic Coatings by Microwave and UV Irradiations}

Initial experiments with the help of UV irradiation are attempted to prepare the monolithic coating on the substrate of glass slides using the typical monomers of methacrylic acid (MAA) and butyl methacrylate (BMA), EDMA as the crosslinker, toluene and isooctane as the binary porogens. The polymerization formula for UV irradiation referred to our previous work [22,23]. Table S1 indicates that the final WCAs are changed in the range of $71-111^{\circ}$ for the resulted monolithic surfaces in the absence and presence of porogens. The fact that no superhydrophobic surface is formed is probably attributed to the use of hydrophilic monomer of MAA. Further attempt is carried out under the given condition that the amount of BMA and EDMA used are fixed, selection of both solvents of butanediol and 1-propanol is found to benefit for the forming of hydrophobic surfaces under an open-air condition. From the results in Table 1, it shows that all fabricated monolithic materials of poly(BMA-co-EDMA) are hydrophobic with the WCAs changed in the range of $94^{\circ}-109^{\circ}$.

Table 1. Preparation conditions for the monolithic coatings of poly(BMA-co-EDMA) and their water contact angles (WCAs) using ultraviolet (UV) irradiation.

\begin{tabular}{ccccccc}
\hline & Monomer & Crosslinker & \multicolumn{2}{c}{ Porogen } & $\begin{array}{c}\text { Percentage } \\
\text { of Porogen } \\
(\%)\end{array}$ & $\begin{array}{c}\text { Average } \\
\text { WCAs }\left(^{\circ}\right)\end{array}$ \\
\cline { 2 - 6 } & $\begin{array}{c}\text { BMA } \\
(\mu \mathrm{L})\end{array}$ & $\begin{array}{c}\text { EDMA } \\
(\mu \mathrm{L})\end{array}$ & $\begin{array}{c}\text { Butanediol } \\
(\mu \mathrm{L})\end{array}$ & $\begin{array}{c}\text { 1-Propanol } \\
(\mu \mathrm{L})\end{array}$ & & UV \\
\hline 1 & 600 & 400 & 156 & 244 & 29 & $94^{\circ}$ \\
2 & 600 & 400 & 233 & 367 & 38 & $100^{\circ}$ \\
3 & 600 & 400 & 350 & 550 & 47 & $107^{\circ}$ \\
4 & 600 & 400 & 506 & 794 & 57 & $95^{\circ}$ \\
5 & 600 & 400 & 700 & 1100 & 64 & $109^{\circ}$ \\
6 & 600 & 400 & 1167 & 1833 & 75 & $95^{\circ}$ \\
\hline
\end{tabular}


In order to transform the hydrophobic monolith to superhydrophobic one, further optimization is needed using relatively low volatility, high viscosity and nonpolar porogens so that high quality of the phase separation can be achieved by microwave and UV irradiation [12]. Typically, a monomer mixture containing a 3:2 volume ratio of BMA and EDMA is fixed but the ratio of lauryl alcohol and 1,4-butanediol is changed for the optimization (See Table S2). In all experiments, the volume ratio $(2 / 3)$ of monomer mixture and binary porogens is kept constant. Microwave and UV irradiation are carried out in 5 min and 30 min using polymeric mixtures 1-6, respectively, so that some coatings with superhydrophobicity are obtained on the glass slides. Table S2 summarizes the preparation conditions for the monolithic coatings and their WCAs using microwave and UV irradiation. For mixture 1 without porogenic agent, the formed non-porous polymers of poly(BMA-EDMA) for both microwave and UV irradiation are very hydrophilic. Fortunately, the porous monoliths for mixture 5 are obtained with the largest WCAs of $170^{\circ}$ and $155^{\circ}$, respectively. After further chemical vapor deposition (CVD) using perfluoro-1,1,2,2-tetrahydrooctyltrichlorosilane (FOTS), the WCAs increase and all monolithic surfaces becoming superhydrophobic but mixtures 1 and 6 (see Figure S1). The very high contact angle $\left(>150^{\circ}\right)$ and extremely low roll-off angle $\left(<5^{\circ}\right)$ clearly indicate that all as-prepared porous surfaces fabricated by both approaches are superhydrophobic. It is quite difficult to dispense water droplets on the monolithic surfaces manually using pipettes. From the results, it is evident that the replacement of 1-propanol with lauryl alcohol leads to a well-developed phase separation state.

\subsection{Characterization and Evaluation}

Surface morphology of the glass slides are obtained by using scanning electron microscopy (SEM) in Figure 1a,b. It is observed that very small spherical particles of nanometer size that are generated during phase-separation and agglomerate forming the interconnected network for two approaches. Interestingly, it is clear that the smallest particle size and the distribution of more regular pore size is obtained for mixture 5 irradiated by UV. These irregular agglomerates consisting of nanometer size polymers and their interconnected network give rise to the Cassie-Baxter state by increasing the effective roughness of the surfaces. Fourier transform-infrared spectroscopy (FT-IR) indicates that poly(BMA-co-EDMA) is successfully formed with some typical characteristic peaks (See Figure S2a). The adsorption peak located at $2961 \mathrm{~cm}^{-1}$ is attributed to the stretching/vibration of $-\mathrm{CH}_{3}$ and $-\mathrm{CH}_{2}$, while the $(\mathrm{C}=)$ stretching/vibration of acrylate occurred located at $1735 \mathrm{~cm}^{-1}$. The carbonyl $(\mathrm{C}=\mathrm{O})$ stretching band occurs at 1600 to $1850 \mathrm{~cm}^{-1}$. The band at $1458 \mathrm{~cm}^{-1}$ is attributed to the skeleton vibration peak of benzene ring. Thermogravimetric analysis (TG) indicates that there are $47 \%$ composition lost at a temperature of $240{ }^{\circ} \mathrm{C}$ and the rest lost at a temperature of $400{ }^{\circ} \mathrm{C}$. The former is the unreacted monomer and porogens and the latter is the cracked polymers of poly(BMA-co-EDMA) (See Figure S2b).

The elemental analysis (i.e., chemical characterization) of the monoliths is carried out by using X-ray photoelectron spectroscopy energy (XPS). As shown in Figure S3, the results show that the coating are composed of carbon (weight $\%=90.0$, atomic $\%=83.4$ ), oxygen (weight $\%=10$, atomic $\%=16.6$ ) for microwave-cured surface; and carbon (weight $\%=85.8$, atomic $\%=79.7$ ), oxygen (weight $\%=14.2$, atomic $\%=20.3$ ) for UV-cured surface, respectively, as the main constituents with carbon having the highest percentage.

The surface topography and nanoscale asperities are measured using a 3D surface profilometer (see Figure 1c) for the optimal polymers. The roughness values obtained from the measurements are as follows: average roughness $(\mathrm{Ra})=16 \mathrm{~nm}$, root mean squared roughness $(\mathrm{Rq})=28 \mathrm{~nm}$ for the blank glass slide; $\mathrm{Ra}=903 \mathrm{~nm}, \mathrm{Rq}=1141 \mathrm{~nm}$ for the microwave-cured glass slide; and a comparative $\mathrm{Ra}=2460 \mathrm{~nm}$, $\mathrm{Rq}=3103 \mathrm{~nm}$ for the UV-cured glass slide. Interestingly, thinner coating with a thickness of $11 \mu \mathrm{m}$ for the microwave-cured glass slide led to a larger RAs than the UV-cured coating with $96 \mu \mathrm{m}$ (see Figure S4). 

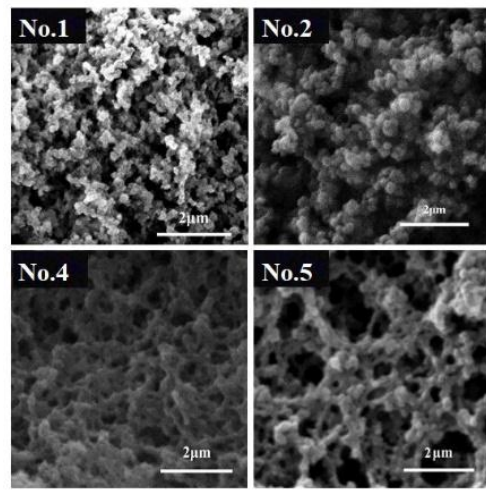

a)
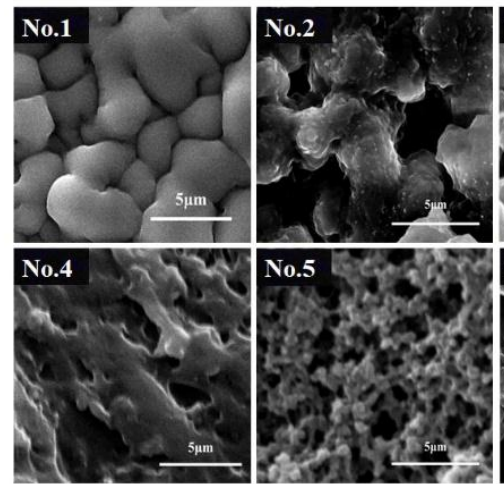

b)

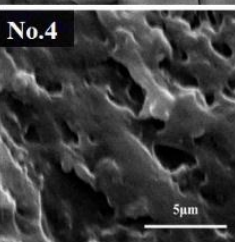

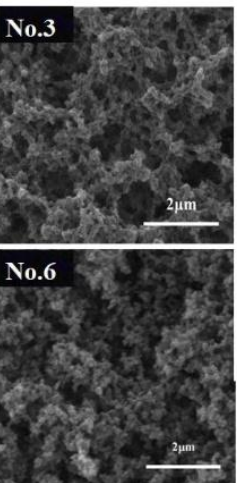

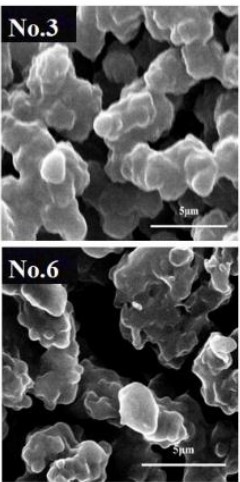

In $-i c$

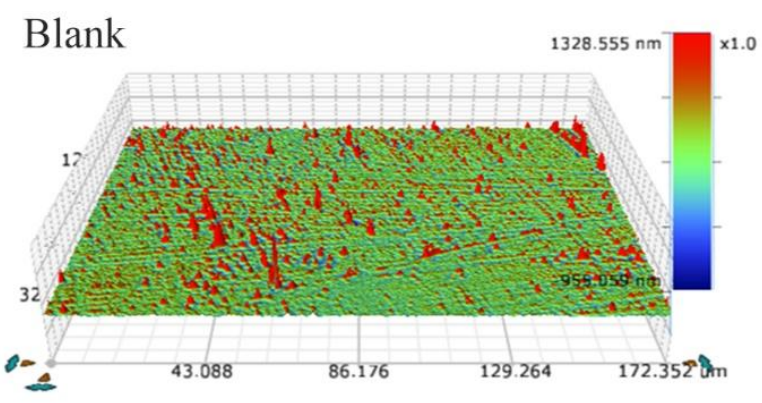

Microwave (No. 5)

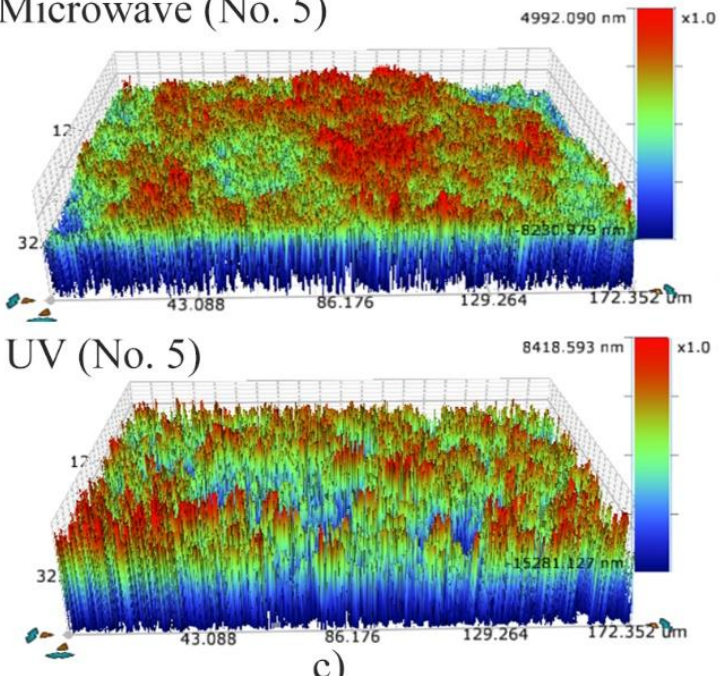

c)

Figure 1. Left: Scanning electron microscopy (SEM) of polymeric coatings fabricated by microwave (a) and UV (b) irradiation; Right: the comparative roughness of the blank glass slide, the optimal microwave-cured coating, the optimal UV-cured coatings (c).

Self-cleaning and water drop bouncing for the microwave-cured coating of mixture 5 were carried out. As shown in Figures S5 and S6 (Videos S1 and S2), the sand particles on the surface are readily removed by spraying with water and the water droplets can bounce up and down on the surface. Meanwhile, the porous polymers ground into particles are pressed on the gloves and water droplet can roll off easily from their faces, respectively (see Video S3).

In order to attain a suitable compromise between high transparency and superhydrophobicity, mixture 5 with the largest WCAs for both methods is selected for the optimization of irradiation time. The results show the monolithic coatings are transformed to superhydrophobic only within 4 min for microwave irradiation but the transparency is hard to further improved (see Figure 2). In contrast, the monolithic coatings are transformed from hydrophobic $\left(\mathrm{WCA}=125^{\circ}\right.$ ) to superhydrophobic within 5-10 min for the UV-curing surface. UV-visible transmittance spectra indicate that longer irradiation time lead to less transparency due to the more monoliths formed. Superhydrophobicity is attained through a combination of suitable roughness and low surface energy material. Whilst transparency is generally achieved by reducing the feature size within the porous polymers because of the scattering of light. Herein, although superhydrophobicity and transparency are generally two conflicting properties that are not easily improved simultaneously, they can be compromised through the optimal experiment. 


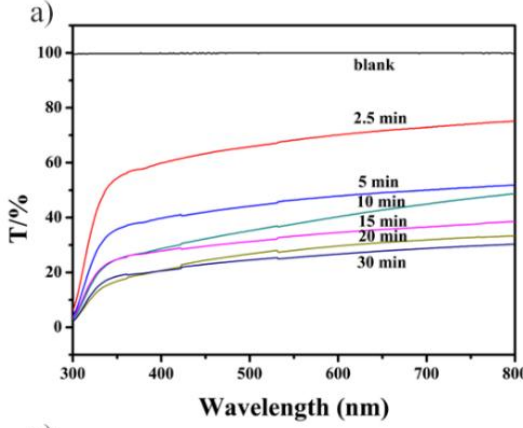

c)

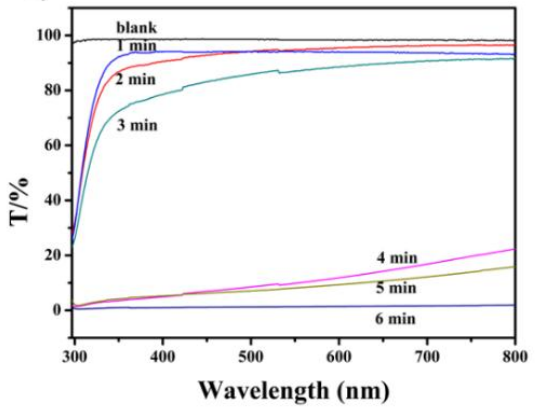

b)

$1 \min 2 \min 3 \min 4 \min 5 \min 6 \min$ T HIST HIST HIST HIST HIST HIST AIIST HIST HIST HIST IIIST HIS THIST HIST HIST HIST HIST HIST HIST HIST HIST HIST I"': III [HIST HIST HIST HIST HIST HIS HIST HIST HIST HIST H THIST HIST HIST HIST HIST HIST HIST HIST H ST HIST HIST HIST HIST HIST HIST HIS HST HIST HST HIST Ho. $-0=0$ d)

$2.5 \mathrm{~min} 5 \mathrm{~min} 10 \mathrm{~min} 15 \mathrm{~min} 20 \mathrm{~min} 30 \mathrm{~min}$ HIST HIST HIST HIST HIST HIST HIST HIST HIST HIST HIST HIST HIST HIST HIST HIST HIST HIST HIST HIST HIST HIST H HIST HIST HIST HIST HIST HIST HIST HIST HIST HIST HIST HIST HIST HIST HIST HIST HIST HIST HIST HIST HIST HIST MIST HISTHIST HIST HIS._HIST HIST HIST HIST HIST HIST

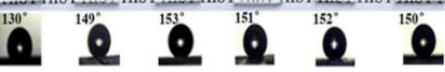

Figure 2. UV-Vis transmittance spectra (a), their relative transparency and their wetting property (b) for the microwave-irradiated coatings of mixture 5. UV-Vis transmittance spectra (c), their relative transparency and their wetting property (d) for the UV-irradiated coatings of mixture 5.

In order to examine the long-term preservation of superhydrophobicity, the glass plates coated with the monolithic coatings are kept in an open-air condition, different extreme environments for 31 days, respectively. As shown in Figure 3, no obvious change of the WCAs is found for both glass plates in the open-air condition, respectively and the surfaces still remain superhydrophobic in one month. The WCAs decrease gradually after both glass plates are immersed more than 10 days in aqueous solutions of $\mathrm{pH}=1, \mathrm{pH}=14$ and $3.5 \% \mathrm{NaCl}$ but all monolithic coatings still remain hydrophobic after immersion in 31 days, respectively.

a)

c)
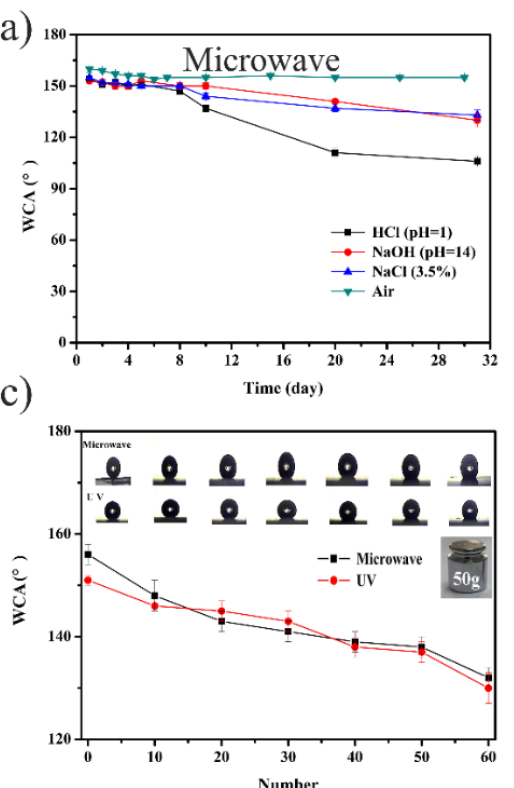

b)

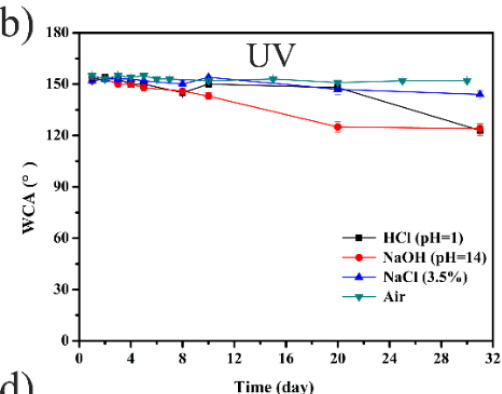

d)

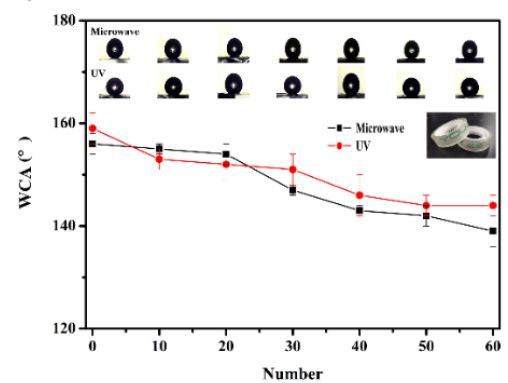

Figure 3. The change of WCAs for both monolithic coatings in air, acid, base and salt for 31 days (a,b), repeatedly abrasion (c) and peel-off (d) experiments for both monolithic coatings, respectively. 
Durability of the coating is further tested against abrasion on the plastic desk with a load of $50 \mathrm{~g}$, wherein the surfaces are abraded for a length of $10 \mathrm{~cm}$, back and forth. Furthermore, the monolithic coatings are drawn repeatedly against a polyester wipe under a pressure of $10 \mathrm{kPa}$ (using dead weight). For all the tests, the WCAs are measured after each 10 consecutive cycles and plotted in Figure 3c,d. Compared to those original states, the final decrease of WCAs is less than $15 \%$ after the abrasion and peel-off tests for 60 cycles, respectively.

In addition, the mechanical stability is also tested by bringing the as-prepared glass slides under a low velocity water jets and letting water drops impinge two typical monolithic coatings from $2 \mathrm{~cm}$ $(0.6 \mathrm{~m} / \mathrm{s})$ and $10 \mathrm{~cm}(1.4 \mathrm{~m} / \mathrm{s})$, respectively (See Table S3 and Figure S7). For the coatings irradiated by microwave, no obvious decrease of WCAs is found after 35,100 and 27,000 drop impacts, with a falling height of $2 \mathrm{~cm}$ and $10 \mathrm{~cm}$, respectively. The final WCAs are $152^{\circ}$ and $142^{\circ}$ accordingly. In contrast, a gradual decrease of WCAs is found for the coatings irradiated by UV with the final WCAs of $124^{\circ}$ and $117^{\circ}$ but still remain hydrophobic. From the results of chemical and mechanical properties, the ruggedness for both optimal polymers on the glass slides are better or close to some previous reports [24-27].

Likewise, other thin substrates were spread in two glass slides for the preparation of monolithic coating including the filter papers, aluminum coil, copper foil and poly tetra fluoroethylene (PTFE) film. Photos of water droplets of acid, base and salt on their surfaces are presented, respectively (see Figure S8). It should be noted these monolithic coatings are fabricated by UV irradiation. Metal foils must be inhibited for the irradiation in order to avoid the combustion due to the microwave interaction.

\subsection{Application of the Prepared Filter Paper}

One filter paper is attempted to be used as a microfluidic paper-based analytical device ( $\mu$ PADs) for the practical application [28,29]. The filter paper is firstly placed on a plastic support with 8 designed holes (holes of $4 \mathrm{~mm}$ in diameter) followed by dropping some polymerization solution of mixture 5, a UV-transparent cover plate with 8 opposite holes is then covered on the plastic support. After UV irradiation about $30 \mathrm{~min}$, the filter paper modified by porous polymers with 8 hydrophilic dots is obtained but water droplets roll off easily on other regions of the whole filter paper except for 8 dots. It is attributed to that the solution dropped on the region with 8 holes leaked or evaporated without the support of the baseplate and effective shield of the upside cover. These hydrophilic dots can be used for introduction of standards and real sample for colorimetric detection. Griess reagent for the detection of nitrite is prepared with $50 \mathrm{mM}$ sulfanilamide and $10 \mathrm{mM}$ naphthalene ethylenediamine hydrochloride in methanol solution. Initially, $10 \mu \mathrm{L}$ of this indicator solution is pipetted onto the reaction zones; following by $10 \mu \mathrm{L}$ different concentrations of nitrite solutions are then added. A magenta azo compound is thus formed. The color results are analyzed by taking photos with a Huawei P10 smartphone and process with the Photoshop program (see Figure 4). These generated magenta stains become stronger upon increasing the nitrite concentration in the range of 0-0.4 mM. In general, RGB (red, green, blue) colors are the three major determinants. The images that are collected by the smartphone are digitized into RGB color coordinates and show non-monotonic behavior with light wavelength and intensity [30]. After these captured colors are transformed to the grey values using greyscale mode with the help of Photoshop software, data are imported into Origin (version 7.5) to obtain linear correlation between grey intensity (GI) and nitrate concentration (C). Herein, a linear correlation of GI $=-194.6 \mathrm{C}+142.6$ is obtained with a correlation coefficient of 0.9896 and a detection limit of $0.04 \mathrm{mM}$ for the standard nitrite assay. These results are comparable with the data reported by the previous report $[28,29,31]$, which is potentially used for the nitrate detection of real samples. 


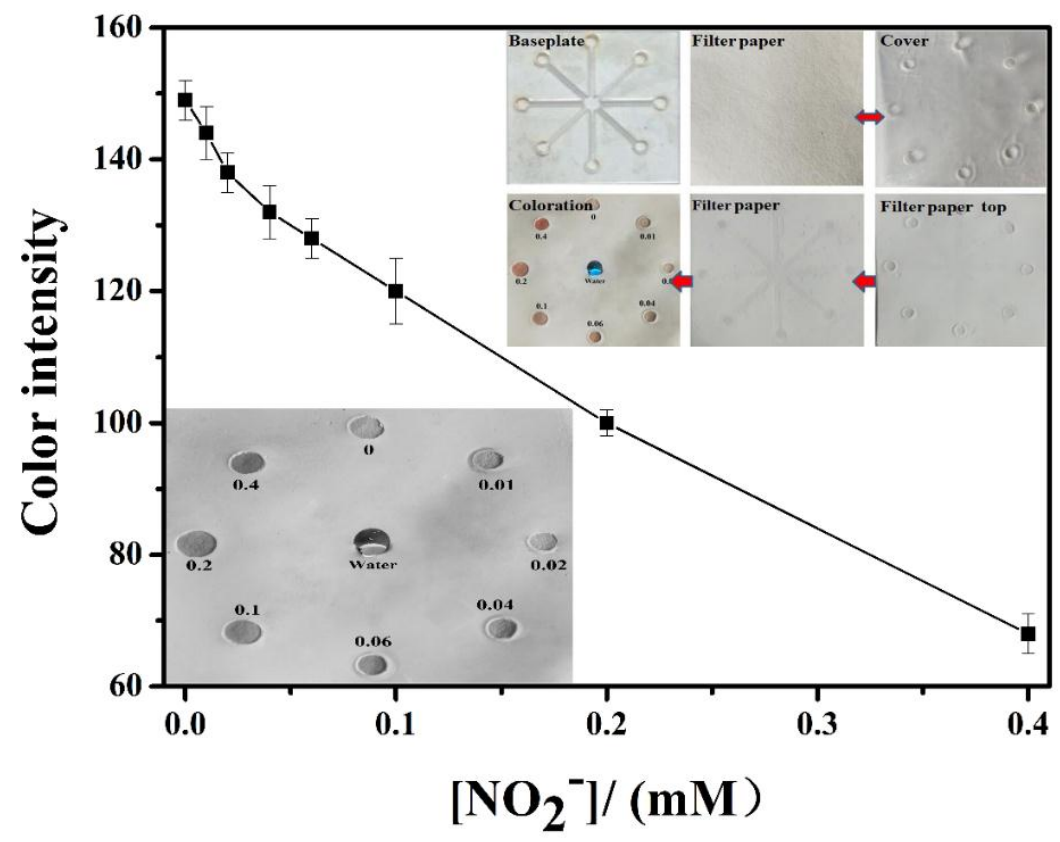

Figure 4. Colorimetric assays fabricated on the substrates of superhydrophobic filter papers. Nitrite assay based on the Griess reaction; the inset shows the prepared procedures (upside) and the analyte concentration in $\mathrm{mM}$ (downside), respectively.

\section{Conclusions}

Two approaches of microwave and UV irradiation are comparatively attempted for the fabrication of monolithic coatings on the glass slide and filter paper conveniently. One step polymerization can be carried out in $10 \mathrm{~min}$ under an open-air condition for both methods. Both optimal polymers on the glass plates reveal distinct structures of porous network with long-term ruggedness in some extreme conditions. With the help of the microwave irradiation, the fabricated coatings on the glass slides exhibit larger WCAs but less transparency under the optimal conditions. It is believed the porogenic agents with less polarity are responsible for the better superhydrophobicity for the resulted monoliths. Most importantly, the superhydrophobic filter paper with some designed hydrophilic dots are readily patterned by UV photopolymerization and adapted for quantitative (nitrite detection) assays. Extended research with the developed method on different substrates for the creation of other more advanced $\mu$ PADs are currently doing in our laboratory.

Supplementary Materials: The following are available online at http://www.mdpi.com/2079-4991/9/3/411/s1, Scheme S1: Fast and simple fabrication of superhydrophobic coating on the glass slide and filter paper by microwave and UV irradiation, Figure S1: The values of WCAs on the monolithic surface before and after CVD for mixtures 1-6 by UV irradiation, Figure S2: (a) FTIR and (b) TG measurements for the UV-cured monolithic coatings, Figure S3: Determination of XPS for the glass sliders with the help of microwave and UV irradiation. (a) XPS survey for the microwave-irradiated surface, (b) c 1s XPS spectrum, (c) O 1s XPS spectrum; (d) XPS survey for the UV-irradiated surface, (e) c 1s XPS spectrum, (f) O 1s XPS spectrum, Figure S4: Thickness of the fabricated monolithic coatings of mixture 5 on the glass sliders by the microwave and UV irradiation, Figure S5: Self-cleaning for the monolithic coating of mixture 5 irradiated by microwave, Figure S6: Drop bouncing on superhydrophobic surface for the monolithic coating of mixture 5 irradiated by microwave, Figure S7: Testing of the coating's long-term resistance against drop impact, Figure S8: The states of water droplets of acid $(\mathrm{pH}=1$, orange), base $(\mathrm{pH}=14$, blue) and salt $(3.5 \% \mathrm{NaCl}$, yellow) sitting on the (a) glass slider, (b) qualitative filter paper, (c) quantitative filter paper, (d) Cu foil, (e) Al foil, (f) PTFE film. The colors orange, blue and yellow are dyed with azorubin, rhodamine B and lemon yellow, respectively, Table S1: Preparation conditions for the monolithic coatings of poly (MAA-co-EDMA) and the WCAs using UV irradiation, Table S2: Preparation condition of the monolithic coatings fabricated using microwave and UV irradiation and their average WCAs, Table S3: The testing condition and WCAs for the durability of the coating towards long-term drop impact, Video S1 showed the self-cleaning on the superhydrophobic poly(BMA-co-EDMA) porous polymeric surface, Video S2 showed the water repellent property on the glass slider prepared by UV irradiation, Video S3 showed the behavior of water on two different gloves coated with the superhydrophobic poly (BMA-co-EDMA) powders. 
Author Contributions: Conceptualization, Y.-P.Z.; Data curation, P.-P.L. and C.-X.C.; Formal analysis, P.-F.L. and W.-Q.Z.; Funding acquisition, Y.-P.Z., X.-J.L., and L.-B.Q.; Investigation, J.-C.W.; Methodology, P.-P.L.; Project administration, Y.-P.Z.

Funding: Financial support from the National Nature Science Foundation of China (No. 51802082) and the Landmark Innovation Project of Henan Institute of Science and Technology (No. 2015BZ02) and the "Funds for Tai Hang Scholar" of HIST and the Science and Technology Project of Henan Province (No. 142102210047) and the Innovation Team in Henan Province (No. C20150020).

Acknowledgments: Thanks Professor Wen-Xiu Fan for his kindly help in the discussion of superhydrophobic mechanisms.

Conflicts of Interest: The authors declare no conflict of interest.

\section{References}

1. Wang, C.; He, Z.; Xie, X.; Mai, X.; Li, Y.; Li, T.; Zhao, M.; Yan, C.; Liu, H.; Wujcik, E.; et al. Controllable Cross-Linking Anion Exchange Membranes with Excellent Mechanical and Thermal Properties. Macromol. Mater. Eng. 2018, 303, 1700462-1700469. [CrossRef]

2. Cui, X.; Zhu, G.; Pan, Y.; Shao, Q.; Zhao, C.; Dong, M.; Zhang, Y.; Guo, Z. Polydimethylsiloxane-titania nanocomposite coating: Fabrication and corrosion resistance. Polymer 2018, 138, 203-210. [CrossRef]

3. Wang, C.; Mo, B.; He, Z.; Xie, X.; Zhao, C.; Zhang, L.; Shao, Q.; Guo, X.; Wujcik, E.; Guo, Z. Hydroxide ions transportation in polynorbornene anion exchange membrane. Polymer 2018, 138, 363-368. [CrossRef]

4. Sheng, Y.; Yang, J.; Wang, F.; Liu, L.; Liu, H.; Yan, C.; Guo, Z. Sol-gel Synthesized Hexagonal Boron Nitride/Titania Nanocomposites with Enhanced Photocatalytic Activity. Appl. Surf. Sci. 2018, 465, 154-163. [CrossRef]

5. Li, S.; Jasim, A.; Zhao, W.; Fu, L.; Ullah, M.; Shi, Z.; Yang, G. Fabrication of pH-Electroactive Bacterial Cellulose/Polyaniline Hydrogel for the Development of a Controlled Drug Release System. ES Mater. Manuf. 2018, 1, 41-49. [CrossRef]

6. Wang, X.; Yang, J.; Sun, X.; Yu, H.; Yan, F.; Meguellati, K.; Cheng, Z.; Zhang, H.; Yang, Y.-W. Facile surface functionalization of upconversion nanoparticles with phosphoryl pillar [5] arenes for controlled cargo release and cell imaging. Chem. Commun. 2018, 54, 12990-12993. [CrossRef] [PubMed]

7. Chu, Z.; Seeger, S. Superamphiphobic surfaces. Chem. Soc. Rev. 2014, 43, 2784-2798. [CrossRef] [PubMed]

8. Liu, Y.; Moevius, L.; Xu, X.; Qian, T.; Yeomans, J.M.; Wang, Z. Pancake bouncing on superhydrophobic surfaces. Nat. Phys. 2014, 10, 515. [CrossRef] [PubMed]

9. Lu, Y.; Sathasivam, S.; Song, J.; Crick, C.R.; Carmalt, C.J.; Parkin, I.P. Robust self-cleaning surfaces that function when exposed to either air or oil. Science 2015, 347, 1132-1135. [CrossRef] [PubMed]

10. Joung, Y.S.; Buie, C.R. Antiwetting Fabric Produced by a Combination of Layer-by-Layer Assembly and Electrophoretic Deposition of Hydrophobic Nanoparticles. ACS Appl. Mater. Interfaces 2015, 7, 20100-20110. [CrossRef] [PubMed]

11. Lee, M.W.; An, S.; Latthe, S.S.; Lee, C.; Hong, S.; Yoon, S.S. Electrospun Polystyrene Nanofiber Membrane with Superhydrophobicity and Superoleophilicity for Selective Separation of Water and Low Viscous Oil. ACS Appl. Mater. Interfaces 2013, 5, 10597-10604. [CrossRef] [PubMed]

12. Tesler, A.B.; Kim, P.; Kolle, S.; Howell, C.; Ahanotu, O.; Aizenberg, J. Extremely durable biofouling-resistant metallic surfaces based on electrodeposited nanoporous tungstite films on steel. Nat. Commun. 2015, 6, 8649. [CrossRef] [PubMed]

13. Deng, X.; Mammen, L.; Butt, H.-J.; Vollmer, D. Candle Soot as a Template for a Transparent Robust Superamphiphobic Coating. Science 2012, 335, 67-70. [CrossRef] [PubMed]

14. Li, Z.; Wang, B.; Qin, X.; Wang, Y.; Liu, C.; Shao, Q.; Wang, N.; Zhang, J.; Shen, C.; Guo, Z. Superhydrophobic/ Superoleophilic polycarbonate/carbon nanotubes porous monolith for selective oil adsorption from water. ACS Sustain. Chem. Eng. 2018, 6, 13747-13755. [CrossRef]

15. Sun, S.; Zhu, L.; Liu, X.; Wu, L.; Dai, K.; Liu, C.; Shen, C.; Guo, X.; Zheng, G.; Guo, Z. Superhydrophobic Shish-kebab Membrane with Self-cleaning and Oil/Water Separation Properties. ACS Sustain. Chem. Eng. 2018, 6, 9866-9875. [CrossRef]

16. Kang, H.; Cheng, Z.; Lai, H.; Ma, H.; Liu, Y.; Mai, X.; Wang, Y.; Shao, Q.; Xiang, L.; Guo, X.; Guo, Z. Superlyophobic Anti-corrosive and Self-cleaning Titania Robust Mesh Membrane with Enhanced Oil/Water Separation, Separation and Purification Technology. Sep. Purif. Technol. 2018, 201, 193-204. [CrossRef] 
17. Nischang, I.; Causon, T.J. Porous polymer monoliths: From their fundamental structure to analytical engineering applications. TrAC Trends Anal. Chem. 2016, 75, 108-117. [CrossRef]

18. Levkin, P.A.; Svec, F.; Fréchet, J.M.J. Porous Polymer Coatings: A Versatile Approach to Superhydrophobic Surfaces. Adv. Funct. Mater. 2009, 19, 1993-1998. [CrossRef] [PubMed]

19. Porcar, R.; Nuevo, D.; García-Verdugo, E.; Lozano, P.; Sanchez-Marcano, J.; Burguete, M.I.; Luis, S.V. New porous monolithic membranes based on supported ionic liquid-like phases for oil/water separation and homogenous catalyst immobilisation. Chem. Commun. 2018, 54, 2385-2388. [CrossRef] [PubMed]

20. Kato, S.; Sato, A. Micro/nanotextured polymer coatings fabricated by UV curing-induced phase separation: Creation of superhydrophobic surfaces. J. Mater. Chem. 2012, 22, 8613-8621. [CrossRef]

21. Xiao, L.; Li, J.; Mieszkin, S.; Di Fino, A.; Clare, A.S.; Callow, M.E.; Callow, J.A.; Grunze, M.; Rosenhahn, A.; Levkin, P.A. Slippery Liquid-Infused Porous Surfaces Showing Marine Antibiofouling Properties. ACS Appl. Mater. Interfaces 2013, 5, 10074-10080. [CrossRef] [PubMed]

22. Zhang, Y.-P.; Ye, X.-W.; Tian, M.-K.; Qu, L.-B.; Choi, S.-H.; Gopalan, A.I.; Lee, K.-P. Novel method to prepare polystyrene-based monolithic columns for chromatographic and electrophoretic separations by microwave irradiation. J. Chromatogr. A 2008, 1188, 43-49. [CrossRef] [PubMed]

23. Chen, N.; Fan, L.-Q.; Gong, W.-J.; Zhang, Y.-P.; Qu, L.-B.; Lee, K.-P. Rapid Preparation and Characterization of Methacrylate-Based Monoliths for Chromatographic and Electrophoretic Separation. J. Chromatogr. Sci. 2010, 48, 399-405. [CrossRef]

24. Hoshian, S.; Jokinen, V.; Franssila, S. Robust hybrid elastomer/metal-oxide superhydrophobic surfaces. Soft Matter 2016, 12, 6526-6535. [CrossRef] [PubMed]

25. Zhou, H.; Wang, H.; Niu, H.; Gestos, A.; Wang, X.; Lin, T. Fluoroalkyl Silane Modified Silicone Rubber/Nanoparticle Composite: A Super Durable, Robust Superhydrophobic Fabric Coating. Adv. Mater. 2012, 24, 2409-2412. [CrossRef] [PubMed]

26. Boinovich, L.; Emelyanenko, A.M.; Pashinin, A.S. Analysis of Long-Term Durability of Superhydrophobic Properties under Continuous Contact with Water. ACS Appl. Mater. Interfaces 2010, 2, 1754-1758. [CrossRef] [PubMed]

27. Malavasi, I.; Bernagozzi, I.; Antonini, C.; Marengo, M. Assessing durability of superhydrophobic surfaces. Surf. Innov. 2014, 3, 49-60. [CrossRef]

28. Lopez-Ruiz, N.; Curto, V.F.; Erenas, M.M.; Benito-Lopez, F.; Diamond, D.; Palma, A.J.; Capitan-Vallvey, L.F. Smartphone-Based Simultaneous $\mathrm{pH}$ and Nitrite Colorimetric Determination for Paper Microfluidic Devices. Anal. Chem. 2014, 86, 9554-9562. [CrossRef] [PubMed]

29. Zhang, L.; Kwok, H.; Li, X.; Yu, H.-Z. Superhydrophobic Substrates from Off-the-Shelf Laboratory Filter Paper: Simplified Preparation, Patterning, and Assay Application. ACS Appl. Mater. Interfaces 2017, 9, 39728-39735. [CrossRef] [PubMed]

30. Jalal, U.M.; Jin, G.J.; Shim, J.S. Paper-Plastic Hybrid Microfluidic Device for Smartphone-Based Colorimetric Analysis of Urine. Anal. Chem. 2017, 89, 13160-13166. [CrossRef] [PubMed]

31. He, Q.; Ma, C.; Hu, X.; Chen, H. Method for Fabrication of Paper-Based Microfluidic Devices by Alkylsilane Self-Assembling and UV/O3-Patterning. Anal. Chem. 2013, 85, 1327-1331. [CrossRef] [PubMed]

(C) 2019 by the authors. Licensee MDPI, Basel, Switzerland. This article is an open access article distributed under the terms and conditions of the Creative Commons Attribution (CC BY) license (http://creativecommons.org/licenses/by/4.0/). 